\title{
Erratum: Experimental Measurement of the Quantum Metric Tensor and Related Topological Phase Transition with a Superconducting Qubit [Phys. Rev. Lett. 122, 210401 (2019)]
}

\begin{abstract}
Xinsheng Tan, Dan-Wei Zhang, Zhen Yang, Ji Chu, Yan-Qing Zhu, Danyu Li, Xiaopei Yang, Shuqing Song,
\end{abstract} Zhikun Han, Zhiyuan Li, Yuqian Dong, Hai-Feng Yu, Hui Yan, Shi-Liang Zhu๑, and Yang Yu

(Received 25 August 2019; published 11 October 2019)

DOI: 10.1103/PhysRevLett.123.159902

In the Letter, we adopted the method in Ref. [1] to calculate the Euler characteristic number $\chi$ for the Hamiltonian $H_{2}(\mathbf{k})$. However, this method is correct for $|h|<1$, but is incomplete (missing the boundary contribution) when $|h|>1$. The manifold of the ground states for $|h|>1$ is equivalent to a disk since the Bloch vectors $\mathbf{d}$ do not entirely cover the Bloch sphere. Actually, the Bloch vectors just run over a crown of the Bloch sphere 4 times. The two-dimensional compact surface $S$ of the crown with the circle boundary is topologically equivalent to a disk with the Euler characteristic number $\chi(S)=1$. Therefore, the total Euler characteristic number is 4 since $\mathbf{d}$ runs over the crown 4 times. After correcting this error calculation, there are two changes: (i) Fig. 4(b) in the Letter should be replaced by Fig. 1 here; (ii) The Euler characteristic number $\chi$ does not change when crossing the critical points $|h|=1$ and thus cannot be used to characterize the topological phase transition of the model described by the Hamiltonian $H_{2}(\mathbf{k})$. The detailed derivation of the Euler number $\chi$ (after taking into account the boundary contribution when $|h|>1)$ for the Hamiltonian $H_{2}(\mathbf{k})$ is given in Ref. [2].

On the other hand, the denominator $2 f^{3 / 2}$ in Eq. (7) should be replaced by $4 f^{3 / 2}$, that is, Eq. (7) in the Letter should be

$$
\sqrt{\operatorname{det} g}=\alpha^{2}\left|\left(1+h \cos k_{x}\right) \sin k_{x}\right| /\left(4 f^{3 / 2}\right) .
$$

The corrections reported here do not change the central conclusions of the Letter, especially the two methods used to measure the quantum metric tensor are correct and all experimental data were correctly collected in the Letter.

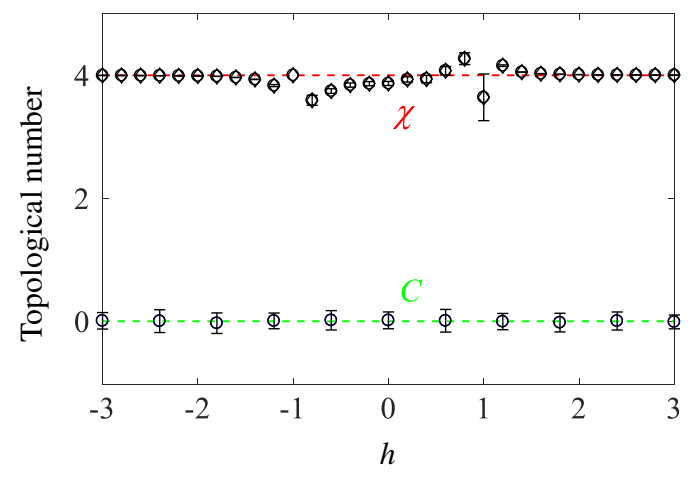

FIG. 1. The Euler characteristic number $\chi$ (after taking into account the boundary contribution) and Chern number $C$ as a function of the parameter $h$. The symbols are measurement data and the dashed lines are theoretical values.

[1] Y.-Q. Ma, S.-J. Gu, S. Chen, H. Fan, and W.-M. Liu, The Euler number of Bloch states manifold and the quantum phases in gapped fermionic systems, Europhys. Lett. 103, 10008 (2013).

[2] Y. Q. Zhu, D. W. Zhang, X. Tan, H. F. Yu, H. Yan, Y. Yu, and S. L. Zhu, Note on "Experimental Measurement of Quantum Metric Tensor and Related Topological Phase Transition with a Superconducting Qubit”, arXiv:1908.06462. 